\title{
Generalized LFT-Based Representation of Parametric Uncertain Models
}

\author{
S. Hecker and A. Varga* \\ German Aerospace Center, DLR - Oberpfaffenhofen, Institute of Robotics and Mechatronics, D-82234 Wessling, Germany
}

In this paper, we introduce a general descriptor-type linear fractional transformation ( $L F T$ ) representation of rational parametric matrices. This is a generalized representation of arbitrary rationally dependent multivariate functions in LFT-form. As applications, we develop explicit LFT-realizations of the transferfunction matrix of a linear descriptor system whose state-space matrices depend rationally on a set of uncertain parameters. The resulting descriptor LFTbased uncertainty models generally have smaller orders than those obtained by using the standard LFT-based modelling approach. An example of an uncertain vehicle model illustrates the capability of the method.

Keywords: Linear Fractional Transformation (LFT); Descriptor Systems; Robust Control; Parametric Uncertainties

\section{Introduction}

In modelling parametric uncertainties in linear systems the linear fractional transformation (LFT) plays an important role. LFT-based representations are useful to model real parametric uncertainties entering rationally in the system matrices. These models are ready to be used in robust control applications like

*E-mail: Andras.Varga@dlr.de

Correspondence to: S. Hecker, German Aerospace Center, DLR Oberpfaffenhofen, Institute of Robotics and Mechatronics, D-82234 Wessling, Germany. Tel.: +49-8153-283374; Fax: +49-8153-281441; E-mail: Simon.Hecker@dlr.de the structured singular value (also called $\mu$ ) [19]. LFTbased models are also useful in representing and manipulating multidimensional systems [3].

The main aspect of LFT-based uncertainty modelling is the generation of low order LFT-representations. Recall that for a partitioned matrix

$$
M=\left[\begin{array}{c|c}
M_{11} & M_{12} \\
\hline M_{21} & M_{22}
\end{array}\right] \in \mathrm{R}^{\left(p_{1}+p_{2}\right) \times\left(m_{1}+m_{2}\right)}
$$

and $\Delta \epsilon \mathbf{R}^{m_{1} \times p_{1}}$, the upper LFT is defined as

$$
\mathcal{F}_{u}(M, \Delta)=M_{22}+M_{21} \Delta\left(I-M_{11} \Delta\right)^{-1} M_{12}
$$

Given a $p_{2} \times m_{2}$ real matrix $G(\boldsymbol{\delta})$ depending rationally on $k$ parameters grouped into the real vector $\boldsymbol{\delta}=\left(\boldsymbol{\delta}_{1}, \ldots, \boldsymbol{\delta}_{k}\right)$, one wants to represent $G(\boldsymbol{\delta})$ as

$$
G(\boldsymbol{\delta})=\mathcal{F}_{u}(M, \Delta),
$$

where $M \in R^{\left(p_{1}+p_{2}\right) \times\left(p_{1}+m_{2}\right)}$ and

$$
\Delta=\operatorname{diag}\left(\delta_{1} I_{r_{1}}, \ldots, \delta_{k} I_{r_{k}}\right)
$$

with $m_{1}=p_{1}=\sum_{i=1}^{k} r_{i}$ representing the order of the LFT-representation (2). The well posedness [19] of the LFT-representation (1) requires that $\left(I-M_{11} \Delta\right)$ is invertible for all $\delta \in \Pi$, where $\Pi$ is the uncertain parameter set defined as

$$
\Pi=\left\{\delta: \delta_{i} \in\left[\delta_{i, \min }, \delta_{i, \max }\right], i=1, \ldots, k\right\} .
$$

Received 19 February 2003; accepted 17 March 2004. Recommended by Y. Ohta and A. Isidori. 
Representing parameter dependent matrices in an LFT-form is basically equivalent to a multidimensional realization problem [3]. There is a basic limitation of realizing arbitrary rational matrices via upper LFTs. Consider the simple case of $G(\delta)=\delta$, which can be immediately realized as

$$
G(\boldsymbol{\delta})=\mathcal{F}_{u}\left(\left[\begin{array}{l|l}
0 & 1 \\
\hline 1 & 0
\end{array}\right], \Delta\right)
$$

with $\Delta=\delta$. However, the expression $G(\boldsymbol{\delta})=1 / \delta$ cannot be directly represented as an upper LFT with $\Delta$ of the form (3). One way to represent $G(\boldsymbol{\delta})=1 / \delta$ as an upper LFT is to use in (5) $\Delta=1 / \delta$. However, $G(\boldsymbol{\delta})=\delta+1 / \delta$ does not have an LFT-representation as both $\delta$ and $1 / \delta$ enter the expression.

In practice, to overcome the above difficulties, a normalization of uncertainties is performed. Assuming, for example, that $\delta \in\left[\delta_{\min }, \delta_{\max }\right]$ and $\delta_{\text {nom }}:=$ $\left(\delta_{\max }+\delta_{\min }\right) / 2 \neq 0$, then with $\delta_{s l}:=\left(\delta_{\max }-\delta_{\min }\right) / 2$ one obtains

$$
\delta=\delta_{\mathrm{nom}}+\delta_{s l} \bar{\delta}
$$

where $\bar{\delta} \in[-1,1]$. With this normalization, we can represent $G(\overline{\boldsymbol{\delta}}):=1 / \delta=1 /\left(\delta_{\text {nom }}+\delta_{s l} \bar{\delta}\right)$ as

$$
G(\overline{\boldsymbol{\delta}})=\mathcal{F}_{u}\left(\left[\begin{array}{c|c}
-\delta_{s l} \delta_{\text {nom }}^{-1} & -\delta_{s l} \delta_{\text {nom }}^{-1} \\
\hline \delta_{\text {nom }}^{-1} & \delta_{\text {nom }}^{-1}
\end{array}\right], \bar{\delta}\right)
$$

Note that this approach is not recommended to be used if $0 \in\left[\delta_{\min }, \delta_{\max }\right]$. One negative aspect of this approach is that the normalization must be performed as a preliminary operation of the LFT-based model generation and this often leads to an increase of the overall order of the LFT-realization [4].

For example, the LFT-realization of $G(\delta)=\delta^{2}$ has order 2, while the LFT-realization of the corresponding normalized expression $G(\bar{\delta})=\delta_{\text {nom }}^{2}+2 \delta_{\text {nom }} \delta_{s l} \bar{\delta}+\delta_{s l}^{2} \bar{\delta}^{2}$ obtained with the object-oriented LFT-realization procedure of [10] has order 3 . This procedure generates an LFT-representation by interconnecting a secondorder LFT-representation for $\delta_{s l}^{2} \bar{\delta}^{2}$ with a first-order LFT-representation for $2 \delta_{\text {nom }} \delta_{s l} \delta$ and the constant term $\delta_{\text {nom }}^{2}$. The result is a third-order LFT-representation. This simple example clearly illustrates that it is desirable to perform the normalization as the last step in any LFT-model generation.

In this paper we introduce a generalized LFTrepresentation which allows the above difficulties to be overcome. The generalized upper LFT is defined with

$$
M=\left[\begin{array}{l|l|l}
M_{10} & M_{11} & M_{12} \\
\hline & M_{21} & M_{22}
\end{array}\right]
$$

as

$$
\mathcal{F}_{u}(M, \Delta)=M_{22}+M_{21} \Delta\left(M_{10}-M_{11} \Delta\right)^{-1} M_{12},
$$

where the submatrix $M_{10}$ is allowed to be generally singular. We call (6) a descriptor LFT, in analogy to the generalized state-space realizations via descriptor systems [5]. For $\Delta$ we assume the more general structure

$$
\Delta=\operatorname{diag}\left(\delta_{0} I_{r_{0}}, \delta_{1} I_{r_{1}}, \ldots, \delta_{k} I_{r_{k}}\right),
$$

where $\delta_{0}$ is a nonzero constant (usually set to 1 ). Note that the standard upper LFT (1) corresponds to $M_{10}=I$ and $r_{0}=0$.

The generalized LFT-representation is a natural extension of the standard LFT-representation and similar representations may also arise in the context of multidimensional system analysis [7].

With this generalized upper LFT we can represent $G(\boldsymbol{\delta})=1 / \delta$ in a descriptor LFT-form as

$$
G(\boldsymbol{\delta})=\mathcal{F}_{u}\left(\left[\begin{array}{rr|rr|r}
0 & 0 & 0 & 1 & 1 \\
0 & 1 & 1 & 0 & 0 \\
\hline & & -1 & 0 & 0
\end{array}\right],\left[\begin{array}{ll}
1 & 0 \\
0 & \delta
\end{array}\right]\right) .
$$

The organization of the paper is as follows. First, we present the basic algebraic properties of the generalized LFT-representations and give explicit formulas for the underlying operations with descriptor LFT-models. These operations form the basis of a general objectoriented LFT-realization procedure for arbitrary rational parametric matrices. We then present results showing that after suitable normalization, the descriptor LFT-representations can be converted into standard LFT-representations. In a separate section we discuss the relationship between our approach to generate LFT-based representations and one that is based on the behavioral setting proposed by D'Andrea in [9]. As an application of the proposed approach, we develop explicit LFT-realizations for the transfer-function matrix of a linear descriptor system whose matrices depend rationally on a set of uncertain parameters. Our result extends those reported in $[12,18]$, where a polynomial parametric dependency of the system matrices is assumed. Finally, we show the capabilities of the proposed method by realizing LFT-representations for a parametric uncertain dynamic vehicle model. 


\section{Algebraic Properties}

Since LFT-based representations are similar to transfer-function matrix representation of linear state-space systems, the basic matrix operations like addition/subtraction, multiplication, transposition, inversion as well as column/row concatenation correspond to similar operations performed on the transfer-function matrices of linear systems. These are operations underlying the methods used to generate LFT-representations of parametric matrices [10]. The following results for descriptor LFT-representations (given without proofs) generalize similar results for standard LFT-representations.

Lemma 2.1. Let $M_{1}, M_{2}$, and $M$ be partitioned matrices

$$
\begin{aligned}
M_{1} & =\left[\begin{array}{l|l|l}
E_{1} & A_{1} & B_{1} \\
\hline & C_{1} & D_{1}
\end{array}\right], \quad M_{2}=\left[\begin{array}{l|l|l}
E_{2} & A_{2} & B_{2} \\
\hline & C_{2} & D_{2}
\end{array}\right], \\
M & =\left[\begin{array}{l|l|l}
E & A & B \\
\hline & C & D
\end{array}\right],
\end{aligned}
$$

and let $\Delta_{1}, \Delta_{2}$ and $\Delta$ be the corresponding uncertainty matrices.

(i) $\mathcal{F}_{u}\left(M_{1}, \Delta_{1}\right) \pm \mathcal{F}_{u}\left(M_{2}, \Delta_{2}\right)=\mathcal{F}_{u}\left(M_{\mathrm{par}}, \Delta_{\mathrm{par}}\right)$ (parallel connection), with

$$
\begin{aligned}
M_{\mathrm{par}} & =\left[\begin{array}{cc|cc|c}
E_{1} & 0 & A_{1} & 0 & B_{1} \\
0 & E_{2} & 0 & A_{2} & \pm B_{2} \\
\hline & & C_{1} C_{2} & D_{1} \pm D_{2}
\end{array}\right], \\
\Delta_{\mathrm{par}} & =\left[\begin{array}{cc}
\Delta_{1} & 0 \\
0 & \Delta_{2}
\end{array}\right] .
\end{aligned}
$$

(ii) $\mathcal{F}_{u}\left(M_{1}, \Delta_{1}\right) \mathcal{F}_{u}\left(M_{2}, \Delta_{2}\right)=\mathcal{F}_{u}\left(M_{\text {ser }}, \Delta_{\text {ser }}\right)$ (series connection), with

$$
\begin{aligned}
M_{\text {ser }} & =\left[\begin{array}{cc|cc|c}
E_{1} & 0 & A_{1} & B_{1} C_{2} & B_{1} D_{2} \\
0 & E_{2} & 0 & A_{2} & B_{2} \\
\hline & & C_{1} & D_{1} C_{2} & D_{1} D_{2}
\end{array}\right], \\
\Delta_{\text {ser }} & =\left[\begin{array}{cc}
\Delta_{1} & 0 \\
0 & \Delta_{2}
\end{array}\right] .
\end{aligned}
$$

(iii) $\left[\mathcal{F}_{u}\left(M_{1}, \Delta_{1}\right) \mathcal{F}_{u}\left(M_{2}, \Delta_{2}\right)\right]=\mathcal{F}_{u}\left(M_{\mathrm{cc}}, \Delta_{\mathrm{cc}}\right)(\mathrm{col}-$ umn concatenation), with

$$
\begin{aligned}
& M_{\mathrm{cc}}=\left[\begin{array}{cc|cc|cc}
E_{1} & 0 & A_{1} & 0 & B_{1} & 0 \\
0 & E_{2} & 0 & A_{2} & 0 & B_{2} \\
\hline & & C_{1} & C_{2} & D_{1} & D_{2}
\end{array}\right], \\
& \Delta_{\mathrm{cc}}=\left[\begin{array}{cc}
\Delta_{1} & 0 \\
0 & \Delta_{2}
\end{array}\right] .
\end{aligned}
$$

(iv) $\left[\mathcal{F}_{u}\left(M_{1}, \Delta_{1}\right)^{\mathrm{T}} \mathcal{F}_{u}\left(M_{2}, \Delta_{2}\right)^{\mathrm{T}}\right]^{\mathrm{T}}=\mathcal{F}_{u}\left(M_{\mathrm{rc}}, \Delta_{\mathrm{rc}}\right)$ (row concatenation), with

$$
M_{\mathrm{rc}}=\left[\begin{array}{cc|cc|c}
E_{1} & 0 & A_{1} & 0 & B_{1} \\
0 & E_{2} & 0 & A_{2} & B_{2} \\
\hline & & C_{1} & 0 & D_{1} \\
& & 0 & C_{2} & D_{2}
\end{array}\right],
$$

$$
\Delta_{\mathrm{rc}}=\left[\begin{array}{cc}
\Delta_{1} & 0 \\
0 & \Delta_{2}
\end{array}\right] .
$$

(v) Suppose $\mathcal{F}_{u}(M, \Delta)$ is a $p \times p$ invertible matrix. Then

$\left(\mathcal{F}_{u}(M, \Delta)\right)^{-1}=\mathcal{F}_{u}\left(M_{\text {inv }}, \Delta_{\text {inv }}\right)$

with

$$
\begin{aligned}
M_{\mathrm{inv}} & =\left[\begin{array}{rr|rr|r}
0 & 0 & D & C & I_{p} \\
0 & E & B & A & 0 \\
\hline & & -I_{p} & 0 & 0 \\
& & 0
\end{array}\right], \\
\Delta_{\mathrm{inv}} & =\left[\begin{array}{ll}
I_{p} & 0 \\
0 & \Delta
\end{array}\right] .
\end{aligned}
$$

If $D$ is invertible, then we can express $M_{\text {inv }}$ and $\Delta_{\text {inv }}$ as

$$
\begin{aligned}
M_{\text {inv }} & =\left[\begin{array}{c|c|c}
E & A-B D^{-1} C & -B D^{-1} \\
\hline & D^{-1} C & D^{-1}
\end{array}\right], \\
\Delta_{\text {inv }} & =\Delta .
\end{aligned}
$$


(vi) Let $Q$ and $Z$ be invertible matrices such that $Z \Delta=\Delta Z$. Then

$\mathcal{F}_{u}(M, \Delta)=\mathcal{F}_{u}(\widetilde{M}, \Delta)$,

where

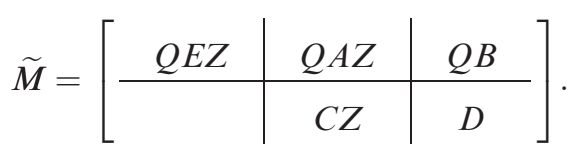

(vii) Consider

$$
\left[\begin{array}{cc}
A(\widetilde{\Delta}) & B(\widetilde{\Delta}) \\
C(\widetilde{\Delta}) & D(\widetilde{\Delta})
\end{array}\right]=\mathcal{F}_{u}\left(\left[\begin{array}{c|c|cc}
\widetilde{E} & \widetilde{A} & \widetilde{B}_{1} & \widetilde{B}_{2} \\
\hline & \widetilde{C}_{1} & \widetilde{D}_{11} & \widetilde{D}_{12} \\
& \widetilde{C}_{2} & \widetilde{D}_{21} & \widetilde{D}_{22}
\end{array}\right], \widetilde{\Delta}\right) .
$$

Then

$$
\mathcal{F}_{u}\left(\left[\begin{array}{c|c|c}
E & A(\widetilde{\Delta}) & B(\widetilde{\Delta}) \\
\hline & C(\widetilde{\Delta}) & D(\widetilde{\Delta})
\end{array}\right], \bar{\Delta}\right)=\mathcal{F}_{u}(M, \Delta)
$$

with

$$
\begin{aligned}
& M=\left[\begin{array}{cc|cc|c}
\widetilde{E} & 0 & \widetilde{A} & \widetilde{B}_{1} & \widetilde{B}_{2} \\
0 & E & \widetilde{C}_{1} & \widetilde{D}_{11} & \widetilde{D}_{12} \\
\hline & & \widetilde{C}_{2} & \widetilde{D}_{21} & \widetilde{D}_{22}
\end{array}\right], \\
& \Delta=\left[\begin{array}{ll}
\widetilde{\Delta} & 0 \\
0 & \bar{\Delta}
\end{array}\right] \text {. }
\end{aligned}
$$

Note that, by using a descriptor LFT-representation of a rational parametric matrix, its inverse (see (v) of Lemma 2.1) can be determined as a descriptor LFT-representation in terms of the original matrices, without any explicit matrix inversion.

It is well known that for any rational parametric matrix $M(\delta)$, we can find a left (right) polynomial fractional representation, with $M(\boldsymbol{\delta})=D^{-1}(\boldsymbol{\delta}) N(\boldsymbol{\delta})$ $\left(M(\boldsymbol{\delta})=\widetilde{N}(\boldsymbol{\delta}) \widetilde{D}^{-1}(\boldsymbol{\delta})\right)$, where $\left.N(\boldsymbol{\delta}), D(\boldsymbol{\delta}) \widetilde{N}(\boldsymbol{\delta}), \widetilde{D}(\boldsymbol{\delta})\right)$ are multivariate polynomial matrices. It is possible to express such fractional representations in terms of the underlying LFT-representations of the factors. The following results are particularly useful when realizing rational parametric matrices in terms of polynomial factorizations.
Lemma 2.2. Let $[N(\boldsymbol{\delta}) D(\boldsymbol{\delta})]=\mathcal{F}_{u}(M, \Delta)$ be defined with

$$
M=\left[\begin{array}{c|c|cc}
E & A & B_{N} & B_{D} \\
\hline & C & D_{N} & D_{D}
\end{array}\right]
$$

and assume that $D(\boldsymbol{\delta})$ is $p \times p$ and invertible. Then

$$
D^{-1}(\boldsymbol{\delta}) N(\boldsymbol{\delta})=\mathcal{F}_{u}\left(M_{l f}, \Delta_{l f}\right)
$$

with

$$
\begin{aligned}
& M_{l f}=\left[\begin{array}{cc|cc|c}
0 & 0 & D_{D} & C & D_{N} \\
0 & E & B_{D} & A & B_{N} \\
\hline & & -I_{p} & 0 & 0 \\
& & & &
\end{array}\right], \\
& \Delta_{l f}=\left[\begin{array}{ll}
I_{p} & 0 \\
0 & \Delta
\end{array}\right] .
\end{aligned}
$$

If $D_{D}$ is invertible we have alternatively

$$
\begin{aligned}
M_{l f} & =\left[\begin{array}{c|c|c}
E & A-B_{D} D_{D}^{-1} C & B_{N}-B_{D} D_{D}^{-1} D_{N} \\
\hline & D_{D}^{-1} C & D_{D}^{-1} D_{N}
\end{array}\right], \\
\Delta_{l f} & =\Delta .
\end{aligned}
$$

Proof. Using (v) and (ii) of Lemma 2.1, we have

$$
D^{-1}(\boldsymbol{\delta}) N(\boldsymbol{\delta})=\mathcal{F}_{u}\left(M_{M}, \Delta_{M}\right),
$$

where

$$
\begin{aligned}
M_{M} & =\left[\begin{array}{c|c|c|c|c}
E_{M} & A_{M} & C_{M} \\
\hline & & C_{M} & D_{M}
\end{array}\right] \\
& :=\left[\begin{array}{lll|ccc|c}
0 & 0 & 0 & D_{D} & C & C & D_{N} \\
0 & E & 0 & B_{D} & A & 0 & 0 \\
0 & 0 & E & 0 & 0 & A & B_{N} \\
\hline & & -I_{p} & 0 & 0 & 0
\end{array}\right], \\
\Delta_{M} & =\left[\begin{array}{ccc}
I_{p} & 0 & 0 \\
0 & \Delta & 0 \\
0 & 0 & \Delta
\end{array}\right] .
\end{aligned}
$$


We now apply a similarity transformation to $M_{M}$, yielding a transformed matrix $\widetilde{M}_{M}$. Consider the transformation matrices $Q$ and $Z$ given by

$$
Q=\left[\begin{array}{ccc}
I_{p} & 0 & 0 \\
0 & I & I \\
0 & 0 & I
\end{array}\right], \quad Z=\left[\begin{array}{ccc}
I_{p} & 0 & 0 \\
0 & I & -I \\
0 & 0 & I
\end{array}\right]
$$

with the identity matrix $I$ of the same size as $\Delta$. It is easy to see that $Z \Delta_{M}=\Delta_{M} Z$, thus applying ( $v i$ ) of Lemma 2.1, we obtain

$$
\begin{aligned}
\tilde{M}_{M}= & {\left[\begin{array}{cc|c|c|c}
Q E_{M} Z & Q A_{M} Z & Q B_{M} \\
\hline & & C_{M} Z & \multicolumn{2}{|c}{D_{M}}
\end{array}\right] } \\
= & {\left[\begin{array}{lll|ccc|c}
0 & 0 & 0 & D_{D} & C & 0 & D_{N} \\
0 & E & 0 & B_{D} & A & 0 & B_{N} \\
0 & 0 & E & 0 & 0 & A & B_{N} \\
\hline & & -I_{p} & 0 & 0 & 0
\end{array}\right] . }
\end{aligned}
$$

By evaluating $\mathcal{F}_{u}\left(\widetilde{M}_{M}, \Delta_{M}\right)$ directly, we see that this expression reduces to $\mathcal{F}_{u}\left(M_{l f}, \Delta_{l f}\right)$.

The result for invertible $D_{D}$ can easily be derived from (10) or it can be proven similarly as done in [2].

The following lemma (given without proof) is the dual result for a right fractional representation.

Lemma 2.3. Let $\left[N^{\mathrm{T}}(\boldsymbol{\delta}) D^{\mathrm{T}}(\boldsymbol{\delta})\right]^{\mathrm{T}}=\mathcal{F}_{u}(M, \Delta)$ be defined with

$$
M=\left[\begin{array}{c|c|c}
E & A & B \\
\hline & C_{N} & D_{N} \\
& C_{D} & D_{D}
\end{array}\right],
$$

and assume that $D(\boldsymbol{\delta})$ is $p \times p$ and invertible. Then

$$
N(\boldsymbol{\delta}) D^{-1}(\boldsymbol{\delta})=\mathcal{F}_{u}\left(M_{\mathrm{rf}}, \Delta_{\mathrm{rf}}\right)
$$

with

$$
\begin{aligned}
M_{\mathrm{rf}} & =\left[\begin{array}{cc|cc|c}
0 & 0 & D_{D} & C_{D} & -I_{p} \\
0 & E & B & A & 0 \\
\hline & & D_{N} & C_{N} & 0
\end{array}\right], \\
\Delta_{\mathrm{rf}} & =\left[\begin{array}{ll}
I_{p} & 0 \\
0 & \Delta
\end{array}\right] .
\end{aligned}
$$

If $D_{D}$ is invertible we have alternatively

$$
\begin{aligned}
M_{\mathrm{rf}} & =\left[\begin{array}{c|c|c}
E & A-B D_{D}^{-1} C_{D} & B D_{D}^{-1} \\
\hline & C_{N}-D_{N} D_{D}^{-1} C_{D} & D_{N} D_{D}^{-1}
\end{array}\right], \\
\Delta_{\mathrm{rf}} & =\Delta .
\end{aligned}
$$

Using the results of this section, we can readily build LFT-representations of an arbitrary rational parametric matrix $G(\boldsymbol{\delta})$ along the lines of the LFTrealization procedure suggested in [10]. This procedure decomposes the realization problem in elementary steps, and starts with the definition of elementary LFT-objects of the form (5) for all distinct parameters $\delta_{i}, i=1, \ldots, k$. Then an LFT-realization of each rational matrix element $g_{i j}(\delta)$ is constructed from the LFT-realizations of its numerator and denominator polynomials (by applying Lemma 2.2). These realizations are constructed using object-oriented realization techniques relying on multivariate polynomial evaluation schemes. The construction of the realization of $G(\boldsymbol{\delta})$ is obtained from the concatenation of the realizations of all its columns, which in their turn follow from the row concatenation of realizations of the elements in each column. Additional symbolic manipulations can contribute significantly in obtaining lower order LFT-realizations. For example, by using Horner or optimal evaluation schemes of polynomials [15], important reduction of orders can be occasionally achieved.

An alternative realization approach of a rational parametric matrix has been proposed in [2]. The LFTrepresentation of $G(\boldsymbol{\delta})$ is built by starting with the calculation of a left (or right) fractional representation $G(\boldsymbol{\delta})=D^{-1}(\boldsymbol{\delta}) N(\boldsymbol{\delta})$ with $D(\boldsymbol{\delta})$ and $N(\boldsymbol{\delta})$ multivariate polynomial matrices. After obtaining $[N(\boldsymbol{\delta}) D(\boldsymbol{\delta})]$ as a standard LFT-representation using the above LFTrealization procedure (possibly jointly with the treedecomposition technique of [4]), the realization of $G(\boldsymbol{\delta})$ follows by employing Lemma 2.2 (or Lemma 2.3). A potential weakness of this approach is the lack of an efficient factorization algorithm with guaranteed minimal degree of denominator factors. Since the degrees of denominators for problems with many parameters and large matrix dimensions tend to be high, the orders of realizations are frequently higher than those resulting from employing the simple approach above.

\section{Normalization}

To obtain at the end a standard LFT-representation ready to be used in standard analysis tools (e.g. in 
$\mu$-analysis), a normalization of the parameters must be usually performed. The main advantage of using generalized LFT-representations is that the obligatory normalization of parameters can be performed at the end of the realization, thus the order of the LFT-representation is not artificially increased by a preliminary normalization of the parameters of the rational parametric matrices. Note that the preliminary normalization can also be avoided when employing the approach of [2]. Here the normalization can be performed after determining the LFT-realization of the factors.

The normalization of parameters amounts to replace each $\delta_{i}$ by $\delta_{i \text {, nom }}+\delta_{i, s} \bar{\delta}_{i}$, where $\delta_{i \text {,nom }}$ and $\delta_{i, s l}$ are such that $\left|\bar{\delta}_{i}\right| \leq 1$, for $i=1, \ldots, k$. To perform the normalization, we have to replace $\Delta$ by $\Delta_{\text {nom }}+\Delta_{s l} \bar{\Delta}$ in the final LFT-representation, where $\Delta_{\text {nom }}, \Delta_{s l}$ and $\bar{\Delta}$ are diagonal matrices with the same structure as $\Delta$ having on the diagonal instead of the $\delta_{i}$, the values of $\delta_{i, \text { nom }}, \delta_{i, s l}$, and $\bar{\delta}_{i}$, respectively. Let us denote the normalized parameter vector by $\bar{\delta}=\left(\bar{\delta}_{1}, \ldots, \bar{\delta}_{k}\right)$. The following result provides formulas to express $G(\overline{\boldsymbol{\delta}})$ in terms of the LFTrepresentation of $G(\boldsymbol{\delta})$.

Lemma 3.1. Let $G(\boldsymbol{\delta})=\mathcal{F}_{u}(M, \Delta)$ with

$$
M=\left[\begin{array}{l|l|l}
E & A & B \\
\hline & C & D
\end{array}\right] .
$$

If $\left(E-A \Delta_{\text {nom }}\right)$ is invertible, then

$$
G(\overline{\boldsymbol{\delta}})=\mathcal{F}_{u}\left(M, \Delta_{\text {nom }}+\Delta_{s l} \bar{\Delta}\right)=\mathcal{F}_{u}(\bar{M}, \bar{\Delta}),
$$

where

$$
\bar{M}=\left[\begin{array}{c|c|c}
I & \bar{A} & \bar{B} \\
\hline & \bar{C} & \bar{D}
\end{array}\right]
$$

with

$$
\begin{aligned}
& \bar{A}=\left(E-A \Delta_{\text {nom }}\right)^{-1} A \Delta_{s l}, \\
& \bar{B}=\left(E-A \Delta_{\text {nom }}\right)^{-1} B, \\
& \bar{C}=C\left(\Delta_{\text {nom }}\left(E-A \Delta_{\text {nom }}\right)^{-1} A+I\right) \Delta_{s l}, \\
& \bar{D}=C \Delta_{\text {nom }}\left(E-A \Delta_{\text {nom }}\right)^{-1} B+D .
\end{aligned}
$$

The order of the resulting normalized standard LFT-representation is the same as the order of the original descriptor LFT-representation. When applying the LFT-realization procedure sketched in the previous section, the resulting LFT-representation $(M, \Delta)$ has the following particular form:

$$
\begin{aligned}
& M=\left[\begin{array}{l|l|l}
E & A & B \\
\hline & C & D
\end{array}\right] \\
& =\left[\begin{array}{cc|cc|c}
0_{r 0} & 0 & A_{11} & A_{12} & B_{1} \\
0 & I & A_{21} & A_{22} & B_{2} \\
\hline & & C_{1} & C_{2} & D
\end{array}\right], \\
& \Delta=\operatorname{diag}\left(I_{r_{0}}, \delta_{1} I_{r 1}, \ldots, \delta_{k} I_{r k}\right)
\end{aligned}
$$

and the corresponding $\Delta_{\text {nom }}$ and $\Delta_{s l}$ will also have the particular forms

$$
\begin{aligned}
\Delta_{\text {nom }} & =\operatorname{diag}\left(I_{r_{0}}, \delta_{1, \text { nom }} I_{r 1}, \ldots, \delta_{k, \text { nom }} I_{r k}\right), \\
\Delta_{s l} & =\operatorname{diag}\left(0_{r_{0}}, \delta_{1, s l} I_{r 1}, \ldots, \delta_{k, s l} I_{r k}\right) .
\end{aligned}
$$

For this particular realization, we have the following specialization of Lemma 3.1, which shows that normalization can lead to a lower order LFT-realization.

Corollary 3.1. Let $G(\boldsymbol{\delta})=\mathcal{F}_{u}(M, \Delta)$ with $\mathrm{M}$ and $\Delta$ given in (11) and (12), respectively, and then let $\Delta=\Delta_{\text {nom }}+\Delta_{s l} \bar{\Delta}$, where $\Delta_{\text {nom }}$ and $\Delta_{\text {sl }}$ have the forms in (13) and (14), respectively. Then $G(\overline{\boldsymbol{\delta}})=\mathcal{F}_{u}(\bar{M}, \bar{\Delta})$ with

$$
\begin{aligned}
& \bar{M}=\left[\begin{array}{c|c|c}
I & \bar{A}_{22} & \bar{B}_{2} \\
\hline & \bar{C}_{2} & \bar{D}
\end{array}\right], \\
& \bar{\Delta}=\operatorname{diag}\left(\bar{\delta}_{1} I_{r 1}, \ldots, \bar{\delta}_{k} I_{r k}\right),
\end{aligned}
$$

where $\bar{A}_{22}, \bar{B}_{2}$, and $\bar{C}_{2}$ are submatrices of the resulting normalized model

$$
\begin{aligned}
\bar{M} & =\left[\begin{array}{c|c|c}
I & \bar{A} & \bar{B} \\
\hline & \bar{C} & \bar{D}
\end{array}\right] \\
& =\left[\begin{array}{cc|cc|c}
I_{r 0} & 0 & \bar{A}_{11} & \bar{A}_{12} & \bar{B}_{1} \\
0 & I & \bar{A}_{21} & \bar{A}_{22} & \bar{B}_{2} \\
\hline & \bar{C}_{1} & \bar{C}_{2} & \bar{D}
\end{array}\right], \\
\bar{\Delta} & =\operatorname{diag}\left(I_{r_{0}}, \bar{\delta}_{1} I_{r 1}, \ldots, \bar{\delta}_{k} I_{r k}\right) .
\end{aligned}
$$

Proof. It follows easily by observing that, as a consequence of the particular structure of $\Delta_{S l}$ in (14), the submatrices $\bar{A}_{11}, \bar{A}_{21}, \bar{C}_{1}$ in (17) are null. 


\section{Relation to the Behavioral Representation}

A behavioral representation for systems with structured uncertainty has been introduced in [9], having the form

$$
z=A \Delta z+B w, \quad 0=C \Delta z+D w .
$$

This description will be referred to as output nulling representation (ONR). In this representation, the vector $z$ is the state and $w$ includes all system variables like inputs, outputs or some so-called latent variables. When manipulating such models there is no need for an a priori choice of input and output variables. In contrast, the explicit descriptor LFT-based representations are input-output type representations. Since conversions between the two representations are straightforward (see below), both representations are suitable to represent arbitrary expressions with rational dependency on uncertain parameters. However, as we will see later, the capabilities of these representations to obtain low order LFT-representations (e.g. suitable for $\mu$-analysis) are quite different.

For the input-output dependence $y=M(\boldsymbol{\delta}) u$, where $\boldsymbol{\delta}=\left(\delta_{1}, \ldots, \delta_{k}\right)$ is a parameter vector, consider the following ONR:

$$
z=A \Delta z+B_{1} y+B_{2} u, \quad 0=C \Delta z+D_{1} y+D_{2} u,
$$

where $B=\left[\begin{array}{ll}B_{1} & B_{2}\end{array}\right]$ and $D=\left[\begin{array}{ll}D_{1} & D_{2}\end{array}\right]$ are partitioned assuming $w=\left[y^{\mathrm{T}} u^{\mathrm{T}}\right]^{\mathrm{T}}$. Assuming that $D_{1}$ is square, an explicit descriptor LFT-representation of $M(\boldsymbol{\delta})$ is the following one:

$$
y=\mathcal{F}_{u}\left(\left[\begin{array}{cc|cc|c}
0 & 0 & D_{1} & C & D_{2} \\
0 & I & B_{1} & A & B_{2} \\
\hline & & -I & 0 & 0
\end{array}\right],\left[\begin{array}{cc}
I & 0 \\
0 & \Delta
\end{array}\right]\right) u .
$$

If $D_{1}$ is invertible, a standard LFT-representation is given by

$y=\mathcal{F}_{u}\left(\left[\begin{array}{c|c|c}I & A-B_{1} D_{1}^{-1} C & B_{2}-B_{1} D_{1}^{-1} D_{2} \\ \hline & -D_{1}^{-1} C & -D_{1}^{-1} D_{2}\end{array}\right], \Delta\right) u$.

Conversely, assume that we have for $M(\boldsymbol{\delta})$ an explicit descriptor LFT-representation (e.g. obtained as described in Section 5.1) of the form

$$
y=\mathcal{F}_{u}\left(\left[\begin{array}{cc|cc|c}
0 & 0 & A_{11} & A_{12} & B_{1} \\
0 & I & A_{21} & A_{22} & B_{2} \\
\hline & & C_{1} & C_{2} & D
\end{array}\right],\left[\begin{array}{cc}
I & 0 \\
0 & \Delta
\end{array}\right]\right) u .
$$

This form can always be achieved by appropriate coordinate transformations. (Note. Due to possible ill-conditioning of these transformations, they are generally not recommended from a numerical point of view to be performed.) An ONR is given by

$$
\begin{aligned}
& z=\left[\begin{array}{rr}
A_{11}+I & A_{12} \\
A_{21} & A_{22}
\end{array}\right]\left[\begin{array}{ll}
I & 0 \\
0 & \Delta
\end{array}\right] z+\left[\begin{array}{ll}
0 & B_{1} \\
0 & B_{2}
\end{array}\right]\left[\begin{array}{l}
y \\
u
\end{array}\right], \\
& 0=\left[\begin{array}{ll}
C_{1} & C_{2}
\end{array}\right]\left[\begin{array}{ll}
I & 0 \\
0 & \Delta
\end{array}\right] z+\left[\begin{array}{ll}
-I & D
\end{array}\right]\left[\begin{array}{l}
y \\
u
\end{array}\right] .
\end{aligned}
$$

From the above relations it follows that the generalized LFT-description introduced in this paper and the ONR are mathematically equivalent formalisms to represent rational parametric matrices.

The basic aspect of generating LFT-based uncertainty descriptions is the efficient representation of interconnected systems. When interconnecting two ONRs, a basic requirement is (see [17]) that the two representations have the same signal space. To ensure this condition, the resulting interconnected system typically contains latent variables and it may be necessary to introduce additional variables to describe the interconnection constraints. The presence of a large number of latent variables (very common for complex ONRs) makes the behavioral approach less suitable for an efficient LFT-based model building. In contrast, standard object-oriented approaches like that described in this paper, produce explicit LFTrepresentations with a "minimal" amount of data. The following simple example will make this aspect clear.

Example 4.1. For the input-output dependence $y=\left(\delta_{1}+\delta_{2}\right) u$ we build an ONR to obtain via (21) an LFT-based representation suitable for $\mu$-analysis. ONRs to represent $y_{i}=\delta_{i} u_{i}$ for $i=1,2$ are given by

$$
z_{i}=u_{i}, \quad 0=\delta_{i} z_{i}-y_{i} .
$$

To represent $y=\left(\delta_{1}+\delta_{2}\right) u$, the interconnection constraints

$$
u_{1}=u_{2}(=u), \quad y=y_{1}+y_{2}
$$


must be fulfilled. To obtain the final ONR, we collect all states in $z=\left[\begin{array}{ll}z_{1} & z_{2}\end{array}\right]^{\mathrm{T}}$ and all variables in $w=\left[\begin{array}{lll}y & y_{1} & u_{1}\end{array}\right.$ $\left.\begin{array}{lll}y_{2} & u\end{array}\right]^{\mathrm{T}}$ and write down the above equations in the standard ONR form

$z=\left[\begin{array}{ll}0 & 0 \\ 0 & 0\end{array}\right]\left[\begin{array}{ll}\delta_{1} & 0 \\ 0 & \delta_{2}\end{array}\right] z+\left[\begin{array}{lllll}0 & 0 & 1 & 0 & 0 \\ 0 & 0 & 0 & 0 & 1\end{array}\right] w$,

$0=\left[\begin{array}{ll}1 & 0 \\ 0 & 1 \\ 0 & 0 \\ 0 & 0\end{array}\right]\left[\begin{array}{ll}\delta_{1} & 0 \\ 0 & \delta_{2}\end{array}\right] z+\left[\begin{array}{rrrrr}0 & -1 & 0 & 0 & 0 \\ 0 & 0 & 0 & -1 & 0 \\ 0 & 0 & 1 & 0 & -1 \\ -1 & 1 & 0 & 1 & 0\end{array}\right] w$

with $\tilde{y}=\left[\begin{array}{llll}y & y_{1} & u_{1} & y_{2}\end{array}\right]^{\mathrm{T}}$ as output variable and $u$ as input variable, we apply now (21) to obtain the explicit LFT-representation

$$
\left[\begin{array}{c}
y \\
y_{1} \\
u_{1} \\
y_{2}
\end{array}\right]=\mathcal{F}_{u}\left(\left[\begin{array}{cc|cc|c}
1 & 0 & 0 & 0 & 1 \\
0 & 1 & 0 & 0 & 1 \\
\hline & & 1 & 1 & 0 \\
& & 1 & 0 & 0 \\
& & 0 & 0 & 1 \\
& & 0 & 1 & 0
\end{array}\right],\left[\begin{array}{cc}
\delta_{1} & 0 \\
0 & \delta_{2}
\end{array}\right]\right) u .
$$

To obtain the LFT-representation of the inputoutput dependence $y=\left(\delta_{1}+\delta_{2}\right) u$ we simply omit the output equations corresponding to the latent variables $y_{1}, u_{1}, y_{2}$. Note that the resulting LFT-representation is just that one which is obtained by using standard inverse-free LFT-manipulations as those described in this paper.

This simple example shows that because of the presence of latent variables, the ONRs have a certain data redundancy, which is not present in the standard or descriptor type LFT-representations. The direct elimination of latent variables in ONRs is quite involved even for 1D systems (see [6]) and we believe to be an open problem in the general multidimensional case.

\section{LFT-Realization of Parametric Descriptor Systems}

In this section, we illustrate the applicability of the proposed approach to solve complex uncertainty modelling problems. We consider the linear parametric system in descriptor form

$$
\begin{aligned}
E(\boldsymbol{\delta}) \dot{x}(t) & =A(\boldsymbol{\delta}) x(t)+B(\boldsymbol{\delta}) u(t), \\
y(t) & =C(\boldsymbol{\delta}) x(t)+D(\boldsymbol{\delta}) u(t)
\end{aligned}
$$

with $u(t) \in \boldsymbol{R}^{m}, \quad x(t) \in \boldsymbol{R}^{n}, \quad y(t) \in \boldsymbol{R}^{p}$ for $t \geq 0$. We assume that $E(\boldsymbol{\delta}), A(\boldsymbol{\delta}), B(\boldsymbol{\delta}), C(\boldsymbol{\delta}), D(\boldsymbol{\delta})$ depend rationally on the components of the parameter vector $\delta$. $E(\boldsymbol{\delta})$ and $A(\boldsymbol{\delta})$ are square matrices and $E(\delta)$ may be singular, but we assume it has constant rank for all $\delta \in \Pi$.

The transfer function matrix $G(s, \boldsymbol{\delta})$ of the descriptor system (24) is given by

$$
G(s, \boldsymbol{\delta})=C(\boldsymbol{\delta})(s E(\boldsymbol{\delta})-A(\boldsymbol{\delta}))^{-1} B(\boldsymbol{\delta})+D(\boldsymbol{\delta}),
$$

where the pencil $|s E(\boldsymbol{\delta})-A(\boldsymbol{\delta})|$ is assumed to be regular for all values of $\boldsymbol{\delta} \in \Pi$.

We develop a general method to determine an LFT representation $(M, \Delta)$ such that

$$
G(s, \bar{\delta})=\mathcal{F}_{u}(M, \Delta)
$$

with

$$
\begin{gathered}
M=\left[\begin{array}{c|c|c}
E_{M} & A_{M} & B_{M} \\
\hline & C_{M} & D_{M}
\end{array}\right], \\
\Delta=\operatorname{diag}\left(1 / s I_{r_{1}}, \bar{\delta}_{2} I_{r 2}, \cdots, \bar{\delta}_{k} I_{r k}\right) .
\end{gathered}
$$

In this LFT-representation the integration operator $1 / s$ (with $s$ as the Laplace variable) is also included in $\Delta$ by defining $\delta_{1}:=1 / s$.

The above realization problem has been addressed in $[18,12]$ for the particular case when all system matrices depend polynomially on the components of the parameter vector $\boldsymbol{\delta}$. Moreover, in [12] it was assumed, that $E(\boldsymbol{\delta})$ is invertible. In the following, we show that an LFT-realization of $G(s, \overline{\boldsymbol{\delta}})$ can be constructed in the most general case of rational parametric matrices, and without assuming the invertibility of $E(\boldsymbol{\delta})$.

For the efficient realization of $G(s, \overline{\boldsymbol{\delta}})$ as an LFTrepresentation, we can distinguish between two cases: (1) $E(\boldsymbol{\delta})$ general (possibly non-invertible); (2) $E(\boldsymbol{\delta})$ invertible.

\section{1. $E(\delta)$ general}

The LFT-realization of $G(s, \overline{\boldsymbol{\delta}})$ can be built using the following steps:

(1) Use the LFT-realization procedure of Section 2 and apply the normalization to determine 
normalized standard LFT-representations for each system matrix of (24), that is, realize

$$
\begin{aligned}
& A(\bar{\delta})=\mathcal{F}_{u}\left(\left[\begin{array}{c|c|c}
I_{n_{a}} & A_{A} & B_{A} \\
\hline & C_{A} & D_{A}
\end{array}\right], \Delta_{A}\right), \\
& B(\bar{\delta})=\mathcal{F}_{u}\left(\left[\begin{array}{c|c|c}
I_{n_{b}} & A_{B} & B_{B} \\
\hline & C_{B} & D_{B}
\end{array}\right], \Delta_{B}\right)
\end{aligned}
$$

and the same for $C(\overline{\boldsymbol{\delta}}), D(\overline{\boldsymbol{\delta}}), E(\overline{\boldsymbol{\delta}})$. Since these matrices do not depend on $s$, the size of $I_{r_{1}}$ within $\Delta_{A}, \ldots, \Delta_{E}$ is zero.

(2) Construct a standard LFT-representation $G(s, \overline{\boldsymbol{\delta}})=\mathcal{F}_{u}(M(s), \widetilde{\Delta})$ with

$$
\begin{aligned}
M(s) & =\left[\begin{array}{c|c|c}
I & M_{11}(s) & M_{12}(s) \\
\hline & M_{21}(s) & M_{22}(s)
\end{array}\right], \\
\widetilde{\Delta} & =\operatorname{diag}\left(\Delta_{E}, \Delta_{A}, \Delta_{B}, \Delta_{C}, \Delta_{D}\right),
\end{aligned}
$$

and

$$
\begin{aligned}
\widetilde{M}(s)= & {\left[\begin{array}{c|cc}
M_{11}(s) & M_{12}(s) \\
\hline M_{21}(s) & M_{22}(s)
\end{array}\right] } \\
& {\left[\begin{array}{ccccc|c}
A_{E} & 0 & 0 & 0 & 0 & 0 \\
0 & A_{A} & 0 & 0 & 0 & 0 \\
0 & 0 & A_{B} & 0 & 0 & B_{B} \\
0 & 0 & 0 & A_{C} & 0 & 0 \\
0 & 0 & 0 & 0 & A_{D} & B_{B} \\
\hline 0 & 0 & 0 & C_{C} & C_{D} & D_{D}
\end{array}\right]-\left[\begin{array}{c}
s B_{E} \\
-B_{A} \\
0 \\
B_{C} \\
0 \\
\hline D_{C}
\end{array}\right] } \\
& \times\left(s D_{E}-D_{A}\right)^{-1}\left[C_{E} C_{A}-C_{B} 00 \mid-D_{B}\right] .
\end{aligned}
$$

(3) Compute a minimal order descriptor realization for the rational matrix $\widetilde{M}(s)$ (e.g. using the methods of [13], followed by the elimination of non-dynamic modes [16]), as

$$
\widetilde{M}(s)=C^{\prime}\left(s E^{\prime}-A^{\prime}\right)^{-1} B^{\prime}+D^{\prime},
$$

and build the corresponding descriptor LFTrepresentation, that is,

$$
\begin{aligned}
\widetilde{M}(s) & =\mathcal{F}_{u}\left(M^{\prime}, \Delta^{\prime}\right) \\
& =C^{\prime} \Delta^{\prime}\left(E^{\prime}-A^{\prime} \Delta^{\prime}\right)^{-1} B^{\prime}+D^{\prime}
\end{aligned}
$$

with

$$
M^{\prime}=\left[\begin{array}{c|c|c}
E^{\prime} & A^{\prime} & B^{\prime} \\
\hline & C^{\prime} & D^{\prime}
\end{array}\right], \quad \Delta^{\prime}=I_{r_{1}} / s .
$$

(4) Apply (vii) of Lemma 2.1 to obtain

$$
G(s, \overline{\boldsymbol{\delta}})=\mathcal{F}_{u}(M, \Delta)
$$

with

$$
\Delta=\operatorname{diag}\left(\Delta^{\prime}, \widetilde{\Delta}\right) .
$$

(5) Reorder $(M, \Delta)$ such that $\Delta$ is of the form given in (26).

\section{2. $E(\delta)$ invertible}

In the case of an invertible $E(\boldsymbol{\delta})$ we can derive a simpler procedure:

(1) Construct a descriptor LFT-representation, such that

$$
\begin{aligned}
& {\left[\begin{array}{cc|cc}
A(\boldsymbol{\delta}) & B(\boldsymbol{\delta}) & E(\boldsymbol{\delta}) & 0 \\
\hline C(\boldsymbol{\delta}) & D(\boldsymbol{\delta}) & 0 & I_{p}
\end{array}\right]=[N(\boldsymbol{\delta}) \mid D(\boldsymbol{\delta})]} \\
& =\mathcal{F}_{u}\left(\left[\begin{array}{c|c|cc}
\tilde{E} & \tilde{A} & \tilde{B}_{N} & \tilde{B}_{D} \\
\hline & \tilde{C} & \tilde{D}_{N} & \tilde{D}_{D}
\end{array}\right], \Delta\right) .
\end{aligned}
$$

(2) Apply (9) and the normalization to obtain the standard LFT-representation

$$
\begin{gathered}
\mathcal{F}_{u}\left(\left[\begin{array}{c|c|cc}
I & A^{\prime} & B_{1}^{\prime} & B_{2}^{\prime} \\
\hline & C_{1}^{\prime} & D_{11}^{\prime} & D_{12}^{\prime} \\
& & & \\
& C_{2}^{\prime} & D_{21}^{\prime} & D_{22}^{\prime}
\end{array}\right], \bar{\Delta}\right. \\
=\left[\begin{array}{cc}
E(\overline{\boldsymbol{\delta}}))^{-1} A(\overline{\boldsymbol{\delta}}) & (E(\overline{\boldsymbol{\delta}}))^{-1} B(\overline{\boldsymbol{\delta}}) \\
C(\overline{\boldsymbol{\delta}}) & D(\overline{\boldsymbol{\delta}})
\end{array}\right] .
\end{gathered}
$$

(3) Construct $G(s, \overline{\boldsymbol{\delta}})$ as

$$
\begin{aligned}
G(s, \overline{\boldsymbol{\delta}}) & =\mathcal{F}_{u}(M, \Delta) \\
& =\mathcal{F}_{u}\left(\left[\begin{array}{cc|cc|c}
I_{n} & 0 & D_{11}^{\prime} & C_{1}^{\prime} & D_{12}^{\prime} \\
0 & I & B_{1}^{\prime} & A^{\prime} & B_{2}^{\prime} \\
\hline & & D_{21}^{\prime} & C_{2}^{\prime} & D_{22}^{\prime}
\end{array}\right],\left[\begin{array}{cc}
I_{n} / s & 0 \\
0 & \bar{\Delta}
\end{array}\right]\right) .
\end{aligned}
$$


(4) Reorder $(M, \Delta)$ such that $\Delta$ is of the form given in (26).

The main advantage of this simpler LFT-realization procedure is that we can apply the symbolic preprocessing techniques of $[4,15]$ to the concatenated symbolic matrix $[N(\boldsymbol{\delta}) \mid D(\boldsymbol{\delta})]$ (see step 1), which contains all matrices of the system. Hence, it is expected that the resulting LFT-realization is of lower order than an LFT-representation, which is realized using the more general procedure of Section 5.1, where each system matrix is realized separately.

\section{Vehicle Model Example}

The proposed LFT-realization method of Section 5.2 is used to compute an LFT-representation of a parametric automotive model presented in [1]. This model describes the lateral motion of a vehicle with an elevated center of gravity for which a robust controller has been designed in [1] to avoid rollover of the vehicle for the whole range of parameter variations. The linearized single-track model is a linear parametric state-space system in descriptor form (24), with

$$
E(\boldsymbol{\delta})=\left[\begin{array}{cccc}
1 & 0 & 0 & 0 \\
0 & m_{1}+m_{2} & 0 & -m_{2} h \\
0 & 0 & 38571 & 0 \\
0 & -m_{2} h & 0 & 24201+m_{2} h^{2}
\end{array}\right]
$$

$A(\boldsymbol{\delta})$
$=\left[\begin{array}{cccc}0 & 0 & 0 & 1 \\ 0 & -1365000 \frac{\mu}{v} 70920 \frac{\mu}{v}-\left(m_{1}+m_{2}\right) v & 0 \\ 0 & 70920 \frac{\mu}{v} & -4070017.8 \frac{\mu}{v} & 0 \\ -457000+9.81 m_{2} h & 0 & m_{2} h v & -100267.6\end{array}\right]$,

$$
\begin{aligned}
B(\boldsymbol{\delta}) & =\left[\begin{array}{llll}
0 & 582000 \mu & 1134900 \mu & 0
\end{array}\right]^{\mathrm{T}}, \\
C & =I_{4}, \quad D=0 .
\end{aligned}
$$

The vehicle consists of a chassis with mass $m_{1}$ and a roll body with mass $m_{2}$, which is mounted above the chassis (see Fig. 1). The center of gravity $C G_{1}$ of the chassis is assumed to be in the road plane. The system state vector is $x=\left[\begin{array}{llll}\phi & v_{y} & r & \dot{\phi}\end{array}\right]^{\mathrm{T}}$, where $\phi$ is the angle of the roll body, $v_{y}=\beta v$ the approximated lateral speed and $r$ the yaw rate. The steering angle $\delta_{f}$ is taken as input. The uncertain parameters are $m_{1}, m_{2}$, $h, v, \mu$, where $h$ is the height of the center of gravity

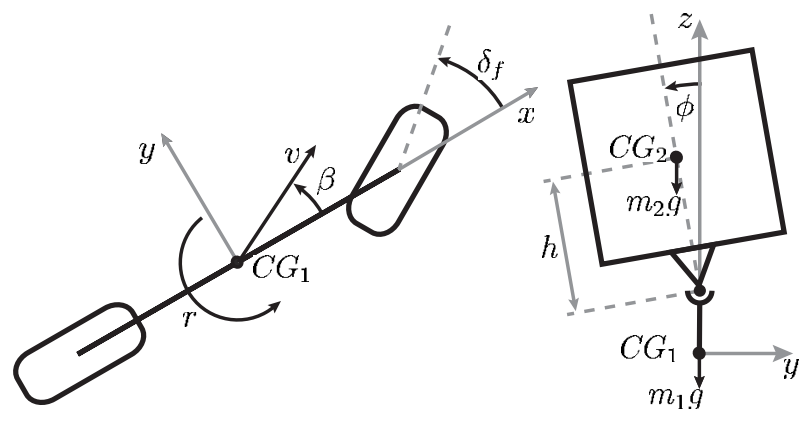

Fig. 1. Single-track vehicle model with roll body.

of the roll body $C G_{2}$ above the roll axis, $v$ is the vehicle velocity and $\mu$ is the road adhesion coefficient. We assume that the uncertain parameters can be expressed as

$$
\begin{aligned}
m_{1} & =1813\left(1+0.05 \bar{\delta}_{2}\right), \\
m_{2} & =12487\left(1+0.2 \bar{\delta}_{3}\right), \\
h & =1.15\left(1+\frac{1}{3} \bar{\delta}_{4}\right), \\
\nu & =\frac{60}{3.6}+\frac{40}{3.6} \bar{\delta}_{5}, \\
\mu & =0.55+0.45 \bar{\delta}_{6}
\end{aligned}
$$

with $\left|\bar{\delta}_{i}\right| \leq 1, i=2, \ldots, 6$, being the normalized uncertainties.

We generated two LFT-representations for the transfer-function matrix $G(s, \overline{\boldsymbol{\delta}})$ of the vehicle model by using two different realization approaches. To determine the first realization we reduced the descriptor system to a standard system by inverting explicitly $E(\boldsymbol{\delta})$. The resulting equivalent parametric standard state-space model is described by the quadruple of matrices $(\widetilde{A}(\boldsymbol{\delta}), \widetilde{B}(\boldsymbol{\delta}), C, D)$, where

$$
\begin{gathered}
\widetilde{A}(\boldsymbol{\delta}):=E^{-1}(\boldsymbol{\delta}) A(\boldsymbol{\delta}), \\
\widetilde{B}(\boldsymbol{\delta}):=E^{-1}(\boldsymbol{\delta}) B(\boldsymbol{\delta}) .
\end{gathered}
$$

After performing the normalization of $\widetilde{A}(\boldsymbol{\delta})$ and $\widetilde{B}(\boldsymbol{\delta})$ we computed an LFT-representation of the parametric system matrix

$$
\left[\begin{array}{cc}
\widetilde{A}(\overline{\boldsymbol{\delta}}) & \widetilde{B}(\overline{\boldsymbol{\delta}}) \\
C & D
\end{array}\right]
$$

along the lines of the LFT-realization procedure of [10]. Finally, we performed step 3 in Section 5.2 to determine the LFT-realization of $G(s, \overline{\boldsymbol{\delta}})$. The second 
Table 1 : Resulted block structures.

\begin{tabular}{llcr}
\hline No. & Reduction & $\left\{r_{1}, r_{2}, r_{3}, r_{4}, r_{5}, r_{6}\right\}$ & \multicolumn{1}{c}{$r$} \\
\hline 1 & No & $\{4,29,42,40,10,9\}$ & 134 \\
1 & Yes & $\{4,13,26,26,3,3\}$ & 75 \\
2 & No & $\{4,2,7,6,6,6\}$ & 31 \\
2 & Yes & $\{4,1,2,2,3,2\}$ & 14 \\
\hline
\end{tabular}

LFT-representation of $G(s, \overline{\boldsymbol{\delta}})$ was calculated by simply performing all realization steps described in Section 5.2.

In addition, for both LFT-representations we repeatedly applied the exact $1-\mathrm{d}$ order reduction of [14] and finally one exact $n$-d order reduction [8]. Table 1 presents the resulting block structure of the uncertainty matrix $\Delta=\operatorname{diag}\left(\frac{1}{s} I_{r 1}, \bar{\delta}_{2} I_{r 2}, \ldots, \bar{\delta}_{6} I_{r_{6}}\right)$ and the total order $r=\sum_{i=1}^{6} r_{i}$ of $\Delta$ for the resulting LFT-representations.

It can be observed in Table 1 that the orders of the initial LFT-realizations for the two realization approaches differ substantially (compare 134 and 31) and a significant difference remains even after applying multidimensional order reduction techniques (compare 75 with 14). The main causes for these huge differences are the explicit symbolic inversion performed to reduce the descriptor system to a standard one and the performed normalization in the initial phase. The elements of the resulting state and input matrices have very complex expressions (e.g. quotients of multivariate polynomials up to orders 23 ), which led to a resulting LFT-realization of considerably higher order. Therefore, besides avoiding the preliminary normalization of uncertain parameters, a second "good practice" for constructing low order LFT-realizations is to also avoid, if possible, explicit symbolic inversions.

Interestingly, the resulting LFT-model of order 14 computed with the proposed approach, appears to be of the least possible order. This can be easily checked by computing the least order partial LFT-realizations with respect to each uncertain parameter. For this particular model, the resulting orders appears to be just the achieved multiplicities in the corresponding blocks of $\Delta$. For inspection purposes the resulting LFT-representations together with the symbolic vehicle model can be downloaded in MATLAB format. $^{1}$

\section{Conclusion}

We proposed a general descriptor system representation based LFT-realization technique for rational parametric matrices. With this approach, we can completely avoid the normalization of the parameters as a preliminary step of the LFT-realization. Therefore, it is generally expected that the resulting LFTrepresentations are of lower order than equivalent representations generated with standard system based LFT-realization methods. Since the proposed method is based on elementary LFT-manipulations it can easily be automated.

In addition, the descriptor system based LFTrealization approach allows to directly derive LFTrepresentations from linear parametric state space systems in descriptor form, which is a usual representation for physical systems. In the proposed procedure, no preliminary symbolic matrix manipulation, like explicit inversion of $E(\boldsymbol{\delta})$ is necessary and even systems with non-invertible $E(\boldsymbol{\delta})$ can be easily handled.

The existing MATLAB LFR-toolbox [11] for the realization of standard LFT-representations has been extended to also handle descriptor LFTrepresentations. Together with reliable numerical tools for handling descriptor systems available in the MATLAB descriptor system toolbox [14] and with symbolic preprocessing techniques for parametric system matrices of $[4,15]$, we have a very promising approach to efficiently generate low order LFTrepresentations of uncertain physical systems.

\section{References}

1. Ackermann $\mathbf{J}$ et al. Robust control. The parameter space approach. In Communications and control engineering, 2nd edn. Springer-Verlag Berlin, October 2002

2. Belcastro CM. Uncertainty modeling of real parameter variations for robust control applications. PhD thesis, University of Drexel, US, December 1994

3. Bose NK. Applied multidimensional systems theory. Van Nostrand-Reinhold Company, Princeton, NJ, 1982

4. Cockburn JC, Morton BG. Linear fractional representation of uncertain systems. Automatica 1997; 33(7): $1263-1271$

5. Dai L. Singular control systems. Number 118 in Lecture Notes in Control and Information Sciences. SpringerVerlag, Berlin, Heidelberg, 1989

6. D'Andrea R. $H_{\infty}$ optimal interconnections. Syst Control Lett 1997; 32: 313-322

7. D'Andrea R, Dullerud GE. Distributed control design for spatially interconnected systems. IEEE Trans Automat Control 2003; 48(9):

8. D'Andrea R, Khatri S. Kalman decomposition of linear fractional transformation representations and minimality. In: Proceedings of the American control conference, pp 3557-3561, Albulquerque, New Mexico, 1997

\footnotetext{
${ }^{1}$ http://www.robotic.dlr.de/ hecker/lft/vehicle.html
} 
9. D'Andrea R, Paganini F. Interconnection of uncertain behavioral systems for robust control. In: Proceedings of the 32nd conference on decision and control, San Antonio, TX, 1993, pp 3642-3647

10. Lambrechts P, Terlouw J, Bennani S, and Steinbuch M. Parametric uncertainty modeling using LFTs. In: Proceedings of the American control conference, San Francisco, CA, 1993, pp 267-272

11. Magni JF. Linear fractional representations with a toolbox for use with MATLAB. Department of Systems Control and Flight Dynamics, ONERACERT, Toulouse, France, December 2001

12. Sugie T, Kawanishi M. $\mu$-analysis/synthesis based on exact expression of physical parameter variations. In: Proceedings of the European Control Conference, Rome, Italy, 1995, pp 159-164

13. Varga A. Computation of irreducible generalized state-space realizations. Kybernetika 1989; 26: 89-106
14. Varga A. A descriptor systems toolbox for MATLAB. In: Proceedings of the CACSD'2000 Symposium, Anchorage, Alaska, 2000

15. Varga A, Looye G. Symbolic and numerical software tools for LFT-based low order uncertainty modeling. In: Proceedings of the CACSD'99 Symposium, Kohala Coast, Hawaii, 1999

16. Verghese G, Lévy B, Kailath T. A generalized statespace for singular systems. IEEE Trans Automat Control 1981; 26: 811-831

17. Willems JC. Paradigms and puzzles in the theory of dynamical systems. IEEE Trans Automat Control 1991; 36(3): 259-294

18. Zerz E. Linear fractional representations of polynomially parametrized descriptor systems. In: Proceedings of the 3rd Portuguese Conference on Automatic Control (Controlo 98), 1998

19. Zhou K, Doyle JC, Glover K. Robust and optimal control. Prentice Hall, Englewood Cliffs, NJ, 1996 